\title{
ILeHCSA: an internet of things enabled smart home automation scheme with speech enabled controlling options using machine learning strategy
}

\author{
Nismon Rio Robert ${ }^{1 *}$, Anija Starry B ${ }^{1}$, F. Jabez Samuel ${ }^{2}$, K. Kala ${ }^{3}$, J. Jenif Preethi ${ }^{4}$, M. Sathish \\ Kumar $^{5}$ and M. Edison ${ }^{6}$ \\ Department of Computer Science, Christ University, Bangalore, India ${ }^{1}$ \\ Department of Information Technology, Bishop Heber College (Autonomous), Tiruchirappalli, Tamil Nadu, India ${ }^{2}$ \\ Department of Computer Science, Nachiappa Swamigal Arts and Science College, Karaikudi, Tamil Nadu, India ${ }^{3}$ \\ Department of Computer Science, J.J. college of Arts and Science (Autonomous), Pudukkottai, Tamil Nadu, India ${ }^{4}$ \\ Department of Computer Applications, Madurai Kamaraj University, Madurai, Tamil Nadu, India ${ }^{5}$ \\ Department of Computer Science, Patrician College of Arts and Science, Chennai,Tamil Nadu, India ${ }^{6}$
}

Received: 24-August-2021; Revised: 20-December-2021; Accepted: 22-December-2021

(C)2021 Nismon Rio Robert et al. This is an open access article distributed under the Creative Commons Attribution (CC BY) License, which permits unrestricted use, distribution, and reproduction in any medium, provided the original work is properly cited.

\begin{abstract}
Nowadays, communication schemes and the related automation logics have improved drastically, and people are moving from classical to intelligent applications. This naturally raises the growth ratio of the automation industry and enables researchers to work accordingly. The field of automation is essential in specific unavoidable environments such as hospitals, industrial units, individual residences, disaster areas, etc. In this paper, a novel machine-learning enabled speech-based home automation system is designed, called Intelligent Learning-enabled Home Controlling with Speech Assistance (ILeHCSA). This scheme integrates several latest technologies to control the home intelligently, including machine learning, speech assistance technology, and Internet of Things (IoT) support. Based on these advanced technologies, the logic of smart home automation systems has been designed in this approach, and it provides intellectual home controlling options to people. The following are the devices and sensors which are essential to control the electronic devices embedded into the home environment: Node Microcontroller Unit (MCU) Wi-Fi enabled Microcontroller, Relay Unit, Voice Capture Module with Mic, Speech-to-Text (STT) Converter Module, and Global Positioning System (GPS) to identify the location of the device. The machine-learning logic is utilized to provide a statistical analysis of device usage and to provide a clear summary and traces to maintain the device accordingly. These smart technologies can innovatively change the living atmosphere with sufficient support and comfort. The main intention of this paper is to provide a robust home automation system to support people efficiently, especially the people who are physically suffering from illness and the aged ones. The proposed work provides a $96.5 \%$ accuracy ratio when compared with other methods.
\end{abstract}

\section{Keywords}

Home automation, Speech assistance, ILeHCSA, Smart home, IoT, Machine learning.

\section{Introduction}

Intelligent, smart home automation systems are the primary requirements of the current world. Smart home automation systems are critical in maintaining living conditions and efficient and scalable surroundings [1]. The purpose of this research is to establish a consistent, smart home automation scheme that allows users to control the electronic devices in their homes using an intelligent application.

\footnotetext{
*Author for correspondence
} 1695
The smart home controlling and automation system enables voice activated control of household devices by detecting the incoming speech [2]. A novel machine learning scheme captures the voice and processes it to securely identify the person to control the devices. Wireless Communication Technology (WCT) is used to build the smart home automation scheme concerning the Internet of Things assistance [3].

A residence based electronic devices like fans, lights, door sensors, window sensors, air conditioners, and etc. is incorporated into a system that interconnected is then to Internet of Thigs (IoT) enabled controller 
that acts as a bridge between the front end and the remote server end and performs the user orders throughout the residence. The voice based smart home controlling system raises living standards and particularly benefits senior citizens [4]. The capacity to operate electronic home devices, not just from a single location but also from anywhere in the globe is a potential for decades to come. A regular wall switch based controlling scheme is positioned around the residence, making it hard for the user to approach it and control the electronic devices accordingly. This becomes significantly more challenging for the aged and severely handicapped. The smart home automation scheme, which is wirelessly deployed by utilizing IoT enabled technology, is capable of controlling the electrical and electronics-based home appliances over a wide area. As innovation advances, smart home automation schemes become smarter. Most advanced homes are transitioning away from wall-based switches and moving toward a centralized command and control system that includes wirelessly operated switching devices. With Android based application technology, a real time based remotely controlled smart home automation scheme gives an easier solution $[5,6]$.

Android operates over a smart phone application platform that consists of an operating system, processors, and other related applications. Android supports multiple modes of communication, including $\mathrm{WiFi}$ technology, Bluetooth communications, and wireless data transfer over a mobile network and base station support. This includes a plethora of relevant tools and features for developing robust applications. Moreover, Android features a comprehensive collection of capabilities that help programmers work more efficiently and gain a deeper understanding of their implementations.

The main motivation of this research is the simplicity and purpose of adopting such features to the residence to convert the normal home into a wireless smart home. A wireless remote operation is enabled via any smart phone/tablet/Personal Computer (PC) /laptop equipped with the Android operating system $[7,8]$, via a Graphical User Interface (GUI) based on the touch sensitive interface as well as voice assistance. This paper provides an option to customize the device according to the user end, with respect to self-configure and remote assistance-based controlling. In self-operating mode, the Android application is presented with a smart device to control the electronic devices in home through local Wi-Fi enabled communications.

The second remote operation mode provides a facility for users to control the devices from anywhere in the globe through an Android mobile application. However, for the second mode of operation the user needs to have the Android mobile to control the devices. To do this, the Android application software serves as a transmitter, transmitting $\mathrm{ON}$ and OFF orders to a receiver linked to loads, as seen in Figure 1 .

Utilizing the wireless enabled technology, electronic devices can be turned ON /or OFF automatically by activating the appropriate voice commands to switch on/off the receiver end devices.

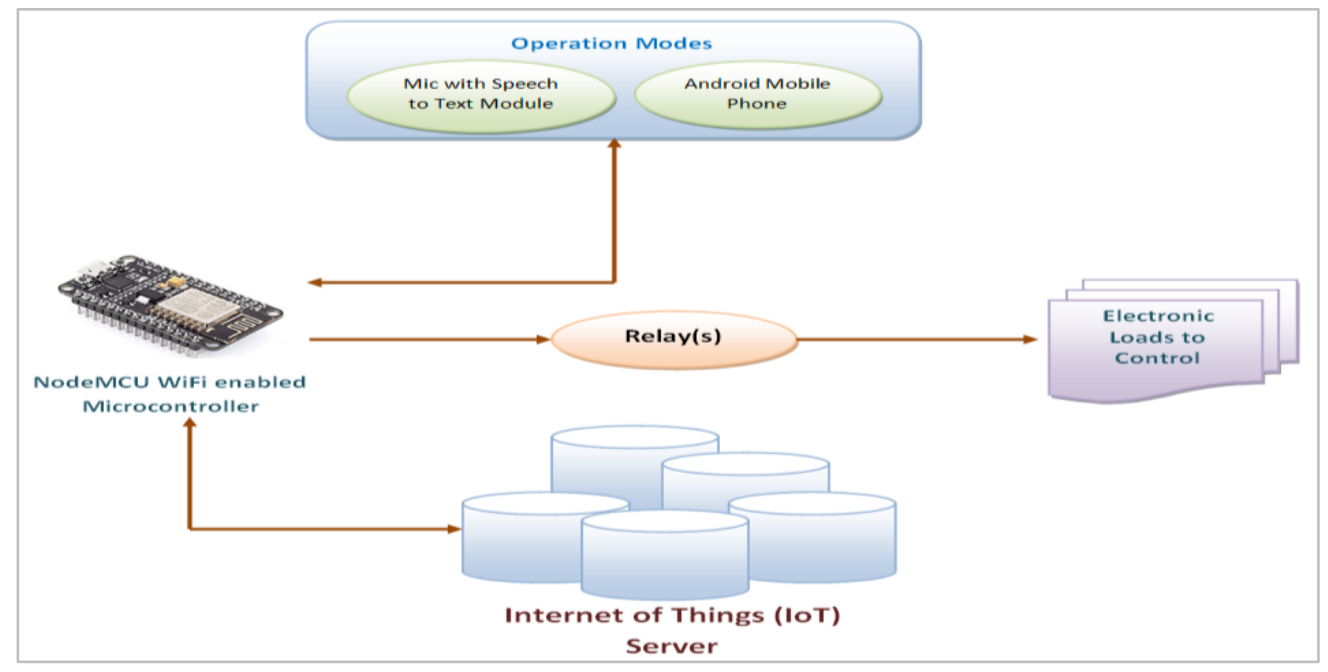

Figure 1 system architectural view 


\subsection{Device operational modes}

The proposed approach utilizes the benefits of dual operation modes such as Mic with Speech to Text (STT) conversion module and the Android based cost efficient remote operation module. Both these modules are efficient enough to operate the homebased electronic devices intelligently. The following summary illustrates the appliances of both these modes in a transparent manner.

\subsection{Mic with STT}

The Mic with STT module contains an inbuilt controller with a pre-programmed voice accumulation module through Mic interfacing. The controller associated with this module consists of a speech recognition system, in which it is deployed by using the python code library called 'Speech Recognition.' This application software called python records and processes voice using the voice identification package and converts the speech into text by using an Application Programming Interface (API) via speech to text environment. Whenever the client speaks, the Mic associated with the device acquires the voice, and the software script developed by using python records the speech in digital format and sends it to the API platform, together with the unique API access code issued the concern to its customers. Once the voice message hits their powerful voice recognition technology, it is converted to text. After that, the text is returned to the script file generated by using python. In the python software script, it is compared to standard instructions for powering ON/OFF equipment. If the keyword resembles the text obtained, the appropriate word ON/OFF is put in the appropriate relay's trigger. A generated python code called iot.py is utilized and it keeps an eye on the content of the files associated with each relay. The scripts generated in python turn the General-Purpose Input-Output (GPIO) pin LOW or HIGH for each relay attached to it according to the script's contents that is either ON or OFF. This mode is especially suitable for the physically challenged and for elderly people.

This mode is significant to capture the voice from a reasonable distance of 5 to 6 feet from the Mic, but if the surrounding noises are less the acquisition ability of the device is more as compared to the mentioned one. This mode of operation is slightly costly compared to another mode called Android-based home automation because this mode consists of several devices such as Mic, STT module with python interfacing, and power adaptors. Due to these requirements, the cost efficiency is less in this mode, but the operating frequency is high enough.

\subsection{Android application}

An Android application-based remote homecontrolling mode offers a provision to customers to control the Home from anywhere in the globe without any restrictions; moreover, this mode is considered to be a cost-effective mode [6]. This is due to the efficiency of mobile phones that are utilized instead of purchasing a separate component to recognize the speech input from the user. This operation scheme accumulates the user's voice input via the google speech recognition tool and accumulates the speech from the client end incorrectly. The accumulated speech values will be processed and converted to text using the Android speech to a text-conversion program, in which the converted textual values are transmitted to the controller via locally enabled Wi-Fi medium.

The accumulated textual values will be analyzed by the controller end for the acquisition of learning principles called Intelligent Learning-enabled Home Controlling with Speech Assistance (ILeHCSA), in which it processes the received voice signals that are accumulated from the authorized person or else it is a fake signal to operate the home electronic components illegally. If the accumulated signals are proper, the respective ON/OFF trigger will be sent to the relay to operate the corresponding devices. Otherwise, this application blocks the user from proceeding further. Based on these features, the proposed approach is significant enough to control the home intelligently from anywhere at any time and without any range restrictions.

The primary objectives of the proposed speechenabled smart home controlling system are summarized as follows:

1. To provide an intelligent home-controlling system with effective operations to people.

2. To support people like physically disabled persons, patients, and old aged people to operate their homes without depending on others.

3. To provide a new methodology to operate the entire home and the associated electronic gadgets based on the user's speech.

4. To provide global operation support with adapting the Internet of Things into the proposed approach.

5. To include the machine-learning strategy to provide a systematic analysis of gadget usage and summarize it through traces, in which it will 
be helpful to maintain the electronic devices in the home accordingly.

These objectives are attained successfully through adding innovative technologies to the conventional home-automation system to transform it into a new manner with the help of learning techniques and IoT associations. The proposed machine-learning model called ILeHCSA is utilized to resolve all the conventional home-automation issues and provides a speech-based home electronic device-controlling system. This logic maintains the operation summary so that the user can easily monitor the summary, and it will be helpful for them in the future.

As regards the rest of this paper, literature review is in section 2, section 3 illustrates the proposed system methodologies in detail, section 4 illustrates the results and discussion portion of the paper and the final section, section 5, illustrates the concept of conclusion and future scope of the proposed paper. All these will be explained in detail in the related section summaries.

\section{Literature review}

Mao et al., [8] proposed a paper related to the identification of ultrasonic sensor-enabled inaudible speech threats on smart home automation schemes. In this paper, the authors emphasize IoT as a vital foundation element and its operational capabilities for the rapidly growing cross-region, intelligent application and diverse cooperative smart building solutions that demand structured collaboration between numerous smart city technologies. Voicecontrolled technologies based on speech-recognition schemes have become one of the foremost similar classifications in new devices.

Dong and Yao [9] proposed a paper related to a secured millimeter wave radar-assisted speaker authentication for the IoT-enabled smart home automation. In this paper, the authors illustrated that voice-assistant devices serve as a communication bridge in an IoT-enabled smart home application. Users can operate smart houses via smart devices. Moreover, smart speakers discuss the implementation of security breaches and data leakages. For instance, hostile players may spoof authorized consumers to submit the home automation based on speech commands. As a result, speech verification has become a vital component of higher security over smart home automation.
Chatterjee et al. [10] proposed a paper related to voice-based emotion assessment in real-time for controlling smart home electronic devices. In this paper, the authors illustrated that artificial intelligence-enabled voice-assisted emotionrecognition scheme is widely utilized in the current market to manage home automation assistants, with many such gadgets in the marketplace. Thus, the purpose of this work is to analyze emotional responses in voice, suggest a method that balances efficiency and sophistication for implementation in consumer products, primarily home-based electronic devices, and demonstrate the study through a realistic live presentation. The paper describes a complete methodology to human voice-assisted emotion recognition. To acquire and identify the feelings connected with the human voice a 1D convolutional layer was developed, and it was built using the Ryerson audio visual database of emotional language and music and the Toronto emotion voice set databases as standardized sources. The suggested methodology achieves prediction performance of 90.48 percent, 95.79 percent, and 94.47 percent in the databases as mentioned earlier.

Su et al. [11] proposed a paper related to a control system for home appliances that is interactive and beneficial to the home to make a smart living experience. In this paper, the authors demonstrate that the easy operation and adaptability of hand-based gesture operations offer immense conceptual and applied promise. The results indicate that the technology works effectively, achieving a classification accuracy of $91 \%$ and completing the controlling function for domestic devices in thirty seconds. The aims of this research are both educational (i) demonstrating the full implementation of methodologies for detection process, handling and information processing and feasible (ii) demonstrating its viability and utilizing commonly produced equipment/software architectures for practical applications and consequently (iii) establishing a framework for effective performance measurement framework to support smart homebased living nature.

Filipe et al. [12] proposed a paper related to applying artificial intelligence and creating a speech-based smart home-controlling system. In this paper, the authors illustrated that the advent of the IoT paradigm had created a clear vision for the technical destiny, enabling the collection and interpretation of data from the surroundings around us through the communication and integration of multiple electronic 
gadgets. Connected devices, in particular, intends to incorporate these gadgets into residences, permitting the mechanization of traditionally manual chores, thereby simplifying daily life and creating a more pleasant space. This research makes the following substantial improvements: (i) architectural design for a home automation control system, (ii) complete implementation of a home automation console and effective communication, (iii) fully accessible set of data of consumer behavior from the smart blinds circumstance and (iv) comparative analysis of offline and online-learning methods.

Venkatraman et al. [13] proposed a model integrating wireless technologies and IoT services to develop a secure smart home automation with artificial intelligence and voice-controlled system. The proposed model is applied to various practical use cases considering factors like integrated voice-based control, security and affordability.

Younis et al. [14] determined a device friendly amicable methodology for home automation. This work alarms the user about gas spillage and door tampering. The methodology focuses on the security factor that automatically sends the alerts and alarms to the user's mobile phone.

Elakkiya et al. [15] discussed web and voice recognition applications to control the electrical appliances at home. Intrusion detection is done by implementing a small-scale prototype using Raspberry pi. The model sends email alerts to the user using an integrated home automation system. Unauthorized person is identified by using a face recognition feature.
Abdulkareem et al. [16] focuses on implementing speech recognition systems integrated with IoT to control door and electrical appliances with raspberrypi elements. Digital Signal Processing technique, speech recognition and hidden Markov model are designed for processing, extraction and high predictive accuracy of the system. The disadvantages of the above-mentioned algorithms and techniques were not quite enough to control the system of the devices. For this reason, ILeHCSA is proposed and to control the system by speech.

\section{ILeHCSA: a proposed algorithm}

ILeHCSA algorithm is proposed, which integrates many latest technologies to intelligently provide a smart home automation system. This proposed approach integrates machine-learning strategies to identify whether the user is a registered person or trying to access the device illegally. This system trains the user voice during the authorization phase and tests the present user voice based on the training samples. When the testing voice sample matches with the trained model, this approach allows the user to proceed further and control the home-based electronic devices through the voice-enabled operations. However, if the input testing voice does not match with the trained sample, it immediately blocks the user from proceeding further. This system consists of numerous smart sensors and gadgets to work with it as depicted in Figure 2. The sensors associated with the proposed approach are Node MCU Wi-Fi-enabled Microcontroller, Relay Unit, Voice Capture Module with Mic, STT Converter Module, and Global Positioning System (GPS) [1719].

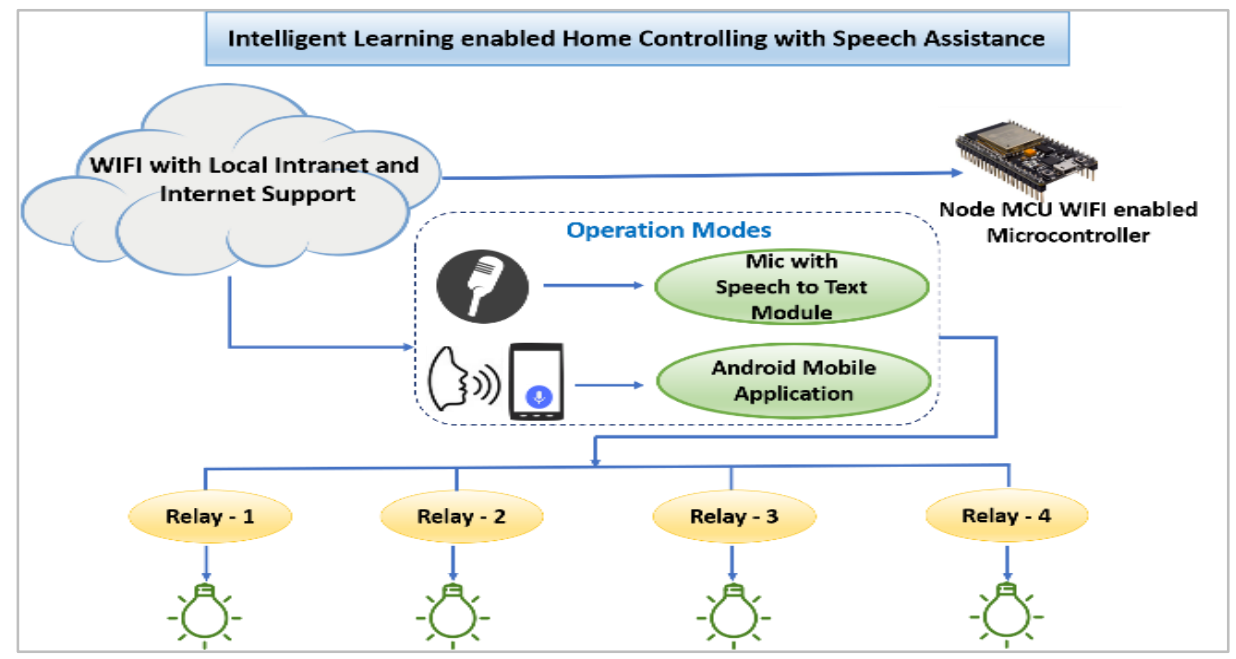

Figure 2 Proposed block diagram 


\author{
Algorithm: Speech Data Accumulation and \\ Controlling \\ Input: User Voice Signals \\ Output: Trigger to Operate the Loads \\ Step-1: Importing the required library to adapt the \\ Wi-Fi features into the code. \\ Pseudo code: Import ESP8266_Wi-Fi.h \\ Step-2: Create an object for Wi-Fi Client class, such \\ as 'client'. \\ Step-3: Create an object for Wi-Fi Server class, such \\ as 'server()' with the specification of transaction byte \\ size. \\ Pseudo code: \\ Wi-Fi_Client client; \\ Wi-Fi_Server server(90); \\ Step-4: Define the Wi-Fi Credentials into the \\ implementation area to connect automatically with \\ the system. \\ Pseudo code: \\ Char SSID<"Username"; \\ Char PWD<"Password";
}

Step-5: Create a SetUp() function to initiate the Wi$F i$ connection and set the baud rate to process the loop accordingly.

Step-6: Create a loop() function and check whether the Wi-Fi credentials are properly initiated or failed to initiate.

Step-7: Gather the voice signals from the URL request parameters and store it into the array unit.

Step-8: Based on the respective array index, the trigger value is grasped from the array.

Step-9: If the trigger value matched with the defined controller, the respective relay will be getting tripped and the load will be ON or OFF accordingly.

\subsection{Relay unit}

A relay is an integrated circuit that functions as a mechanical switch via the use of an electromagnetic function. A relay's primary function is to switch on and off a high-powered connection from a lowerpowered connection [14]. A relay switch is available in various configurations, dimensions, colors, voltage ratings, and characteristics. A relay enables you to switch on or off a circuit with far more voltage and power than a microcontroller can handle. The relay completely isolates the controller's low voltage circuit from the high voltage side operating the loads. The user can activate a relay remotely over $\mathrm{Wi}-\mathrm{Fi}$ from a gadget such as a smartphone, tablet, and computer or activate the relay locally through Wi-Fi. For instance, a Smartphone application can be used to turn on/off-powered household lights. The IoT control relay is a programmable energy circuit with four outlets that enables IoT applications' reliable and safe control scheme. The IoT assisted relay enables the simple regulation of the energy provided to a device via a controller.

\subsection{Voice capture module with Mic}

This module internally consists of voice-capturing Mic, in which it accumulates the user voice through python scripting with Google's support. By using this device, users can speak and control the home electronic gadgets without any interference. This module internally contains the STT module with a programmable controller. The module accumulates the user voice from the Mic and converts it into the text format for validation. The learning approach analyzes the controlling trigger and respective user authentication for the trained dataset model. The validation outcome provides a positive result, which means that the user can trigger the respective load; otherwise, the system blocks the user from controlling the home. This process is very helpful for elderly people and patients.

\subsection{GPS module}

GPS was established to facilitate defense and civilian applications to determine their geographic regions accurately. It is realized utilizing globe orbiting satellites that provide data that allows for measuring the range between both the satellites and the users. The GPS module comprises minuscule Central Processing Unit (CPU) and antennas that acquire information precisely from satellites via specific radio signals, and it will receive timestamps and additional information from each accessible satellite. GPS satellites are equipped with atomic clocks that maintain exact time. The timestamp is included in the satellite's transmission codes so that a receiver may consistently detect when the information was transmitted [15]. This module provides an ability to the proposed approach to maintaining the exact location details of the device with clear traces.

\section{Results and discussion}

The successful placements of the complete installation kit into the residence are tested for ten successive days without any interference or time gap. The accuracy estimations are derived for this application based on the logic and working conditions of the proposed home automation device. The voice is collected from several individuals and tests the proposed scheme accordingly with real-time conditions such as noisy, silent, and moderate environments under dual modes of operations. With all these constraints, the kit's working nature is significant enough and provides a standard accuracy 
ratio of $96.5 \%$ in working. Table 1 illustrates the speech data accumulation ratio over the tested period with success and failure rates. When the number of days was set to 1 , overall data accumulation was $81 \%$, the success rate was $70 \%$, and the failure rate was $11 \%$. Similarly, calculations were done for the various numbers of days. The success rate increased up to $96 \%$ when the total number of days was increased, and the failure rate decreased to $4 \%$.

Table 1 Data accumulation ratio with success and failure ratio analysis

\begin{tabular}{llll}
\hline $\begin{array}{l}\text { No. of } \\
\text { days }\end{array}$ & $\begin{array}{l}\text { Overall } \\
\text { accumulation }\end{array}$ & Success & Failure \\
\hline 1 & $81 \%$ & $70 \%$ & $11 \%$ \\
\hline 2 & $86 \%$ & $80 \%$ & $6 \%$ \\
\hline 3 & $89 \%$ & $84 \%$ & $5 \%$ \\
\hline 4 & $91 \%$ & $86 \%$ & $5 \%$ \\
\hline 5 & $91.2 \%$ & $90 \%$ & $1.2 \%$ \\
\hline 6 & $91.9 \%$ & $89.9 \%$ & $2 \%$ \\
\hline 7 & $92.6 \%$ & $87.6 \%$ & $5 \%$ \\
\hline 8 & $93.06 \%$ & $90.06 \%$ & $3 \%$ \\
\hline
\end{tabular}

\begin{tabular}{llll}
\hline $\begin{array}{l}\text { No. of } \\
\text { days }\end{array}$ & $\begin{array}{l}\text { Overall } \\
\text { accumulation }\end{array}$ & Success & Failure \\
\hline 9 & $94.1 \%$ & $91 \%$ & $4.1 \%$ \\
\hline 10 & $96 \%$ & $92 \%$ & $4 \%$ \\
\hline
\end{tabular}

Figure 3 illustrates the proposed approach speechrecognition accuracy over the tested period in a realtime environment. Moreover, the resulting accuracy is cross-validated by comparing both operation modes, such as Mic with STT module and the Android application-based module. The x-axis indicates the number of days the device is tested, and the y-axis indicates the voice capturing accuracy ratio. On day 1 , Mic with STT exhibits $89 \%$, Android module $91 \%$, and conventional model $81 \%$ accuracy for voice capturing. Similarly, tests are conducted to find the best voice accuracy ratio using Mic with STT, Android module, and conventional model. Consequently, it was found that the Android module had consistently outperformed the other two modules in speech-recognition accuracy.

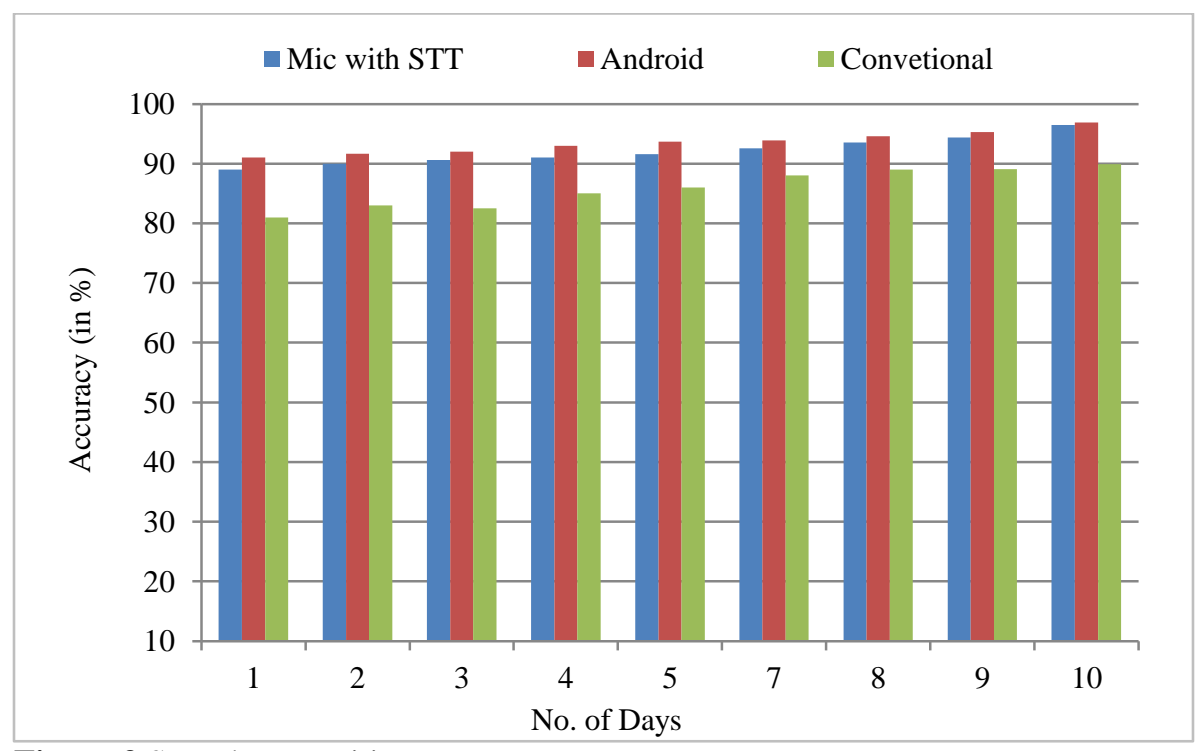

Figure 3 Speech recognition accuracy

Figure 4 illustrates the graphical representation of the proposed approach to data trigger reception accuracy over the tested period in a real-time environment. Moreover, the trigger accuracy ratio is crossvalidated with the comparison of both operation modes such as Mic with STT module as well as the Android application-based module, in which the $\mathrm{x}$ axis indicates the number of days the device is tested and the $y$-axis indicates the triggering accuracy ratio. Trigger reception accuracy is calculated for three modules: Mic with STT, Android, and conventional with the number of days. The results showed that the Android module's trigger accuracy ratio was $92 \%$ to $96 \%$ for days 1 to 10 , declaring that the Android module works better when compared with Mic with STT and conventional models.

Figure 5 illustrates the graphical representation of the proposed approach bit error ratio over the tested period in a real-time environment. Moreover, the resulting bit error ratio is cross-validated by comparing both operation modes, such as Mic with 
STT module and the Android application-based module. The $\mathrm{x}$-axis indicates the number of days the device is tested, and the y-axis indicates the bit error ratio. The graphical representation of the bit error rate analysis indicates that the Android module's bit error rate is less when compared with Mic with STT and conventional models.

\section{Limitations}

The proposed system can be operating according to the control of google voice assistant, in which most people are unaware of using such schemes acceptably. The learning technique requires some time for processing the present service request for the cross-validation of previous requests mentioned in the trace. The logic of deep learning can be applied to the approach to convert the trace verification process simpler.

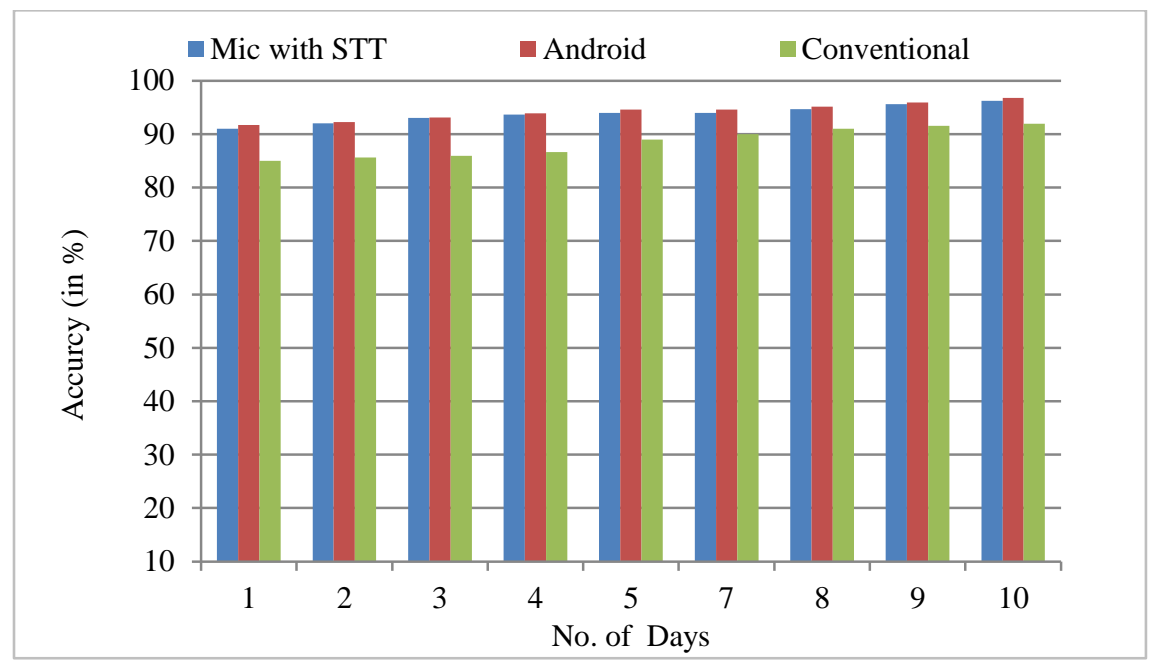

Figure 4 Trigger reception accuracy

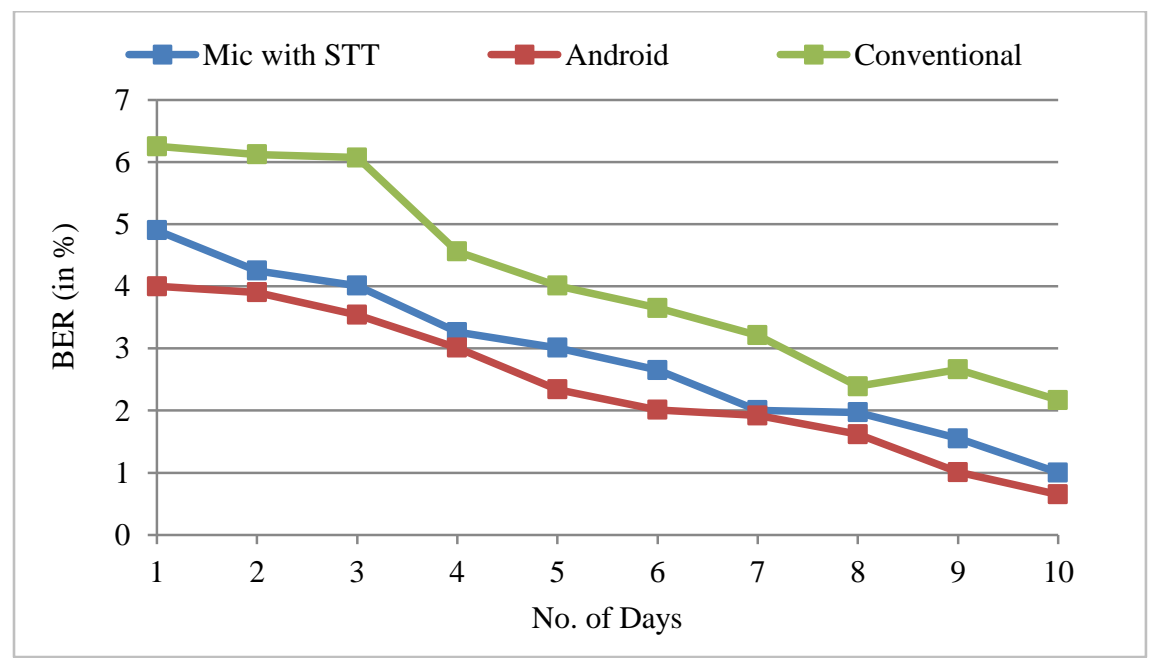

Figure 5 Bit error rate analysis

\section{Conclusion and future scope}

The proposed ILeHCSA is concerned with powerful controlling methodologies. This system provides dual mode controlling options such as Mic-based voice capturing and controlling and android applicationbased smart home controlling. This technology has several innovative devices associated with it to manipulate the automation intelligently. Specifically, the appliance of the GPS module provides robust support to assist the users based on device location exactly concerning latitude and longitude values. This system is more suitable for elderly people and can easily be controlled via Wi-Fi, and especially it is suitable for handicapped people. The IoT appliance 
provides robust features to the proposed approach to operate the device from a remote environment as well. This voice-based smart home automation system is a speech-activated household control strategy that combines an Android application to operate household electronic appliances through WCT. Because no direct interaction with the smart home automation system is necessary, speech-based controlling options provide users with a sense of stability. The Android-assisted smart home application talks with the remote IoT data server through the network through the mobile application. Every Android Smartphone capable of running the smart home automation software is used to download and install it and regulate the smart home automation system. A reduced expensive logic of this smart home automation scheme has been designed, in which the Wi-Fi-enabled controller intelligently performs all computations. By utilizing wireless communication strategies, the suggested solution eliminates the need for additional wiring.

In the future, the work can further be enhanced by adding some hybrid learning approaches to the proposed learning scheme to improve the time efficiency. The AdaBoost logic can be utilized for improving the speech-recognition accuracy.

\section{Acknowledgment}

None.

\section{Conflicts of interest}

The authors have no conflicts of interest to declare.

\section{References}

[1] Noruwana NC, Owolawi PA, Mapayi T. Interactive IoTbased speech-controlled home automation system. In international multidisciplinary information technology and engineering conference 2020 (pp. 1-8). IEEE.

[2] Sravanthi G, Madhuri G, Sharma N, Tiwari A, Kashyap A, Suresh B. Voice recognition application based home automation system with people counter. In international conference on advances in computing, communication control and networking 2018 (pp. 574-8). IEEE.

[3] Chowdhury KD, Choudhury D, Sharmin A, Roy A, Kundu A. Analysis on speech recognition for automation. In international conference for convergence in engineering 2020 (pp. 161-4). IEEE.

[4] Martinez-martin E, Costa A. Assistive technology for elderly care: an overview. IEEE Access. 2021.

[5] Hasan M, Biswas P, Bilash MT, Dipto MA. Smart home systems: overview and comparative analysis. In fourth international conference on research in computational intelligence and communication networks 2018 (pp. 264-8). IEEE.
[6] Khan WM, Zualkernan IA. Sensepods: a zigbee-based tangible smart home interface. IEEE Transactions on Consumer Electronics. 2018; 64(2):145-52.

[7] Nath P, Pati UC. Low-cost android app based voice operated room automation system. In 3rd international conference for convergence in technology 2018 (pp. 14). IEEE.

[8] Mao J, Zhu S, Dai X, Lin Q, Liu J. Watchdog: detecting ultrasonic-based inaudible voice attacks to smart home systems. IEEE Internet of Things Journal. 2020; 7(9):8025-35.

[9] Dong Y, Yao YD. Secure MMWave-radar-based speaker verification for IoT smart home. IEEE Internet of Things Journal. 2020; 8(5):3500-11.

[10] Chatterjee R, Mazumdar S, Sherratt RS, Halder R, Maitra T, Giri D. Real-time speech emotion analysis for smart home assistants. IEEE Transactions on Consumer Electronics. 2021; 67(1):68-76.

[11] Su MC, Chen JH, Arifai AM, Tsai SY, Wei HH. Smart living: an interactive control system for household appliances. IEEE Access. 2021; 9:14897-904.

[12] Filipe L, Peres RS, Tavares RM. Voice-activated smart home controller using machine learning. IEEE Access. 2021; 9:66852-63.

[13] Venkatraman S, Overmars A, Thong M. Smart home automation - use cases of a secure and integrated voicecontrol system. Systems. 2021; 9(4):1-13.

[14] Younis SA, Ijaz U, Randhawa IA, Ijaz A. Speech recognition based home automation system using raspberry $\mathrm{Pi}$ and Zigbee. NFC IEFR Journal of Engineering and Scientific Research. 2018; 5(2):40-5.

[15] Elakkiya E, Shruthi T, Malini GM. IoT based intelligent system for home automation using voice recognition. International Journal of Innovative Research in Electrical, Electronics, Instrumentation and Control Engineering. 2020; 8(6):174-80.

[16] Abdulkareem A, Somefun TE, Chinedum OK, Agbetuyi F. Design and implementation of speech recognition system integrated with internet of things. International Journal of Electrical and Computer Engineering. 2021; 11(2):1796-803.

[17] Andrade SH, Contente GO, Rodrigues LB, Lima LX, Vijaykumar NL, Frances CR. A smart home architecture for smart energy consumption in a residence with multiple users. IEEE Access. 2021; 9:16807-24.

[18] Arzo ST, Naiga C, Granelli F, Bassoli R, Devetsikiotis M, Fitzek FH. A theoretical discussion and survey of network automation for IoT: challenges and opportunity. IEEE Internet of Things Journal. 2021; 8(15):12021-45.

[19] Jacquenet C. Optimized, automated, and protective: an operator's view on future networks. IEEE Transactions on Network and Service Management. 2021; 18(2):1350-9. 


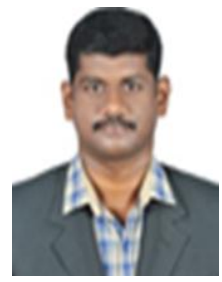

Dr. Nismon Rio Robert is currently working as an Assistant Professor in the Computer Science, CHRIST (Deemed to be University), Bangalore, India, since 2019. He holds a Ph.D. Degree in Computer Science from Bishop Heber College affiliated to Bharathidasan University, Tiruchirappalli, India in 2019. He received financial support from University Grants Commission (UGC) of the research work. His research interest is Route Optimization in Wireless Networks, Mobile Ad-hoc Networks, Internet of Things and other related topics. He has presented 30 papers in National and International conferences/journals. Moreover, he has published 6 Indian Patents in the field of communication technologies. He has delivered technical lectures in various institutions.

Email: nismon.rio@christuniversirty.in

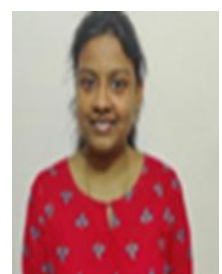

Mrs. Anija Starry B is currently pursuing $\mathrm{PhD}$ at CHRIST (Deemed to be University), Bangalore, India, since June 2021. Her research interest is Data Management in the Internet of Things. She has presented 3 papers in national and international conferences/journals.

Email: anija.starry@res.christuniversity.in

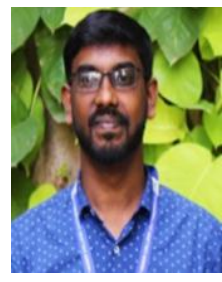

F. Jabez Samuel is currently working as an Assistant Professor in Information Technology, Bishop Heber College (Autonomous), Tiruchirappalli, Tamil $\mathrm{Nadu}$, India. He has attended various conferences and workshops related to his area of research interest. His research interest is Internet of Things and Data Mining. He has published one international article in a reputed journal also he has been continuing his doctoral research program in Government Arts College, Thiruverumbur, which is affiliated to Bharathidasan University.

Email: samueljabez2@gmail.com

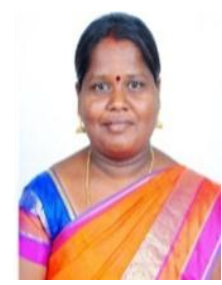

Dr. K. Kala is currently working as an Associate Professor of Computer Science, Nachiappa Swamigal Arts \& Science College, Karaikudi, Tamil Nadu, India. She has 19 years of teaching experience. She has wide experience in administrate level. She has presented 30 papers in national and international conferences/journals. Email: kasinathkala1971@yahoo.co.in

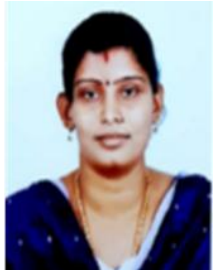

Mrs. J. Jenif Preethi is currently working as an Assistant Professor of Computer Science, J.J. College of Arts $\&$ Science (Autonomous), Pudukkottai, India, since 2020. She Worked from 2010-2012 at St. Joseph's College, Trichy, India. She pursued her M.Sc. Computer Science from Loyola College, Chennai, India in 2008. She holds her M.Phil. from St. Joseph's College, Trichy, India in 2009. Her M.Phil. thesis is based on an Artificial Neural Network with Cognitive behavioural therapy. Her Research interest is to find out new things in technologies. She has presented 4 Papers in National and International Conferences. Moreover, she has published 1 Indian Patent in the field of Biomedical Engineering.

Email: jenifpreethijjc2020@gmail.com

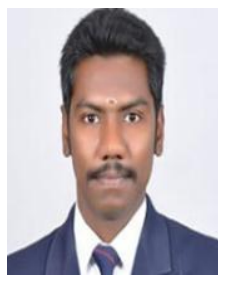

M. Sathish Kumar is a doctoral research scholar in Computer Applications at Madurai Kamaraj University, Madurai, India, His research interest is Internet Security and Cyber Security. He has presented 20 papers in national and international conferences/journals. He has published 1 Indian Patent in the field of communication technology. Email: sathish.friends89@gmail.com

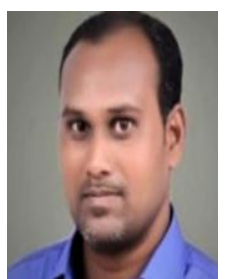

Dr. M. Edison is currently working as an Assistant Professor in Computer Science, Patrician College of Arts and Science, Adyar, Chennai, Tamil Nadu, India. He holds a Ph.D. degree in Computer Science from St. Joseph's College (Autonomous), Trichy affiliated to Bharathidasan University, Trichy, Tamil Nadu, India in 2018. He has published 12 papers in International Journals in Big Data, Sentiment Analysis, Data Mining and Software Metrics. He has published 1 Indian Patent in the field of Robotics.

Email: edi.muthu01@gmail.com

\begin{tabular}{lll}
\multicolumn{2}{l}{ Appendix $\mathbf{1}$} \\
\hline S. No. & Abbreviation & Description \\
\hline 1 & API & Application Programming Interface \\
\hline 2 & GPIO & General-Purpose Input Output \\
\hline 3 & GPS & Global Positioning System \\
\hline 4 & GUI & Graphical User Interface \\
\hline 5 & ILeHCSA & $\begin{array}{l}\text { Intelligent Learning enabled Home } \\
\text { Controlling with Speech Assistance }\end{array}$ \\
\hline 6 & IoT & Internet of Things \\
\hline 7 & PC & Personal Computer \\
\hline 8 & MCU & Microcontroller Unit \\
\hline 9 & STT & Speech to Text \\
\hline 10 & WCT & Wireless Communication Technology
\end{tabular}

